\title{
Abdominal near-infrared spectroscopy in a piglet model of gastrointestinal hypoxia produced by graded hypoxia or superior mesenteric artery ligation
}

\author{
May W Chen ${ }^{1}$, Michael Reyes ${ }^{2}$, Ewa Kulikowicz ${ }^{2}$, Laura Martin ${ }^{3}$, David J Hackam³ ${ }^{3}$ Raymond C Koehler ${ }^{2}$ and Jennifer K Lee ${ }^{2}$
}

BACKGROUND: Abdominal near-infrared spectroscopy (aNIRS) may detect gastrointestinal hypoxia before necrotizing enterocolitis develops. We sought to validate aNIRS during splanchnic hypoxia and hypoperfusion in neonatal piglets.

METHODS: Anesthetized piglets underwent systemic hypoxia or $3 \mathrm{~h}$ superior mesenteric artery (SMA) ligation with aNIRS monitoring.

RESULTS: During progressive hypoxia, gastrointestinal tissue oxyhemoglobin saturation measured by aNIRS decreased linearly with oxyhemoglobin saturation measured directly in the portal vein. Correlation coefficients were 0.94-0.99 in each of 10 piglets, the average regression slope of 0.73 (95\% confidence interval: $0.57,0.89)$ differed from one $(P<0.004)$, and the intercept on the aNIRS axis of $9.5 \%(4.4,14.6)$ differed from zero $(P<0.0025)$. Umbilical venous oxyhemoglobin saturation also correlated strongly with the portal vein oxyhemoglobin saturation $(r=0.83-0.99)$, with a slope not different from one. SMA ligation caused ileal blood flow to decrease by $\sim 50 \%$, and produced a sustained decrease in aNIRS oximetry from approximately 60 to $30 \%$.

CONCLUSION: aNIRS can detect abrupt and sustained gastrointestinal hypoperfusion associated with arterial occlusion in a neonatal model. The highly linear relationship of portal venous oxyhemoglobin saturation with aNIRS and umbilical vein saturation during graded hypoxia implies that these measures can accurately track tissue oxygenation trends over a wide range in individual subjects.

$\mathbf{N}$ eonatal gastrointestinal ischemia and necrotizing enterocolitis (NEC) can have devastating consequences, especially if the diagnosis is delayed. Noninvasive methods that diagnose gastrointestinal hypoxia early might improve outcomes. Near-infrared spectroscopy (NIRS) is a continuous, non-invasive tool that measures regional tissue oxyhemoglobin saturation (1). Changes in NIRS can reflect the changes in tissue perfusion and the risk of ischemic injury $(2,3)$. The gastrointestinal tract is highly vulnerable to injury because blood flow is directed toward other vital organs in critically ill infants (4). Pathologic abdominal processes, such as NEC, intestinal volvulus, or intestinal ischemia are often diagnosed late, when irreversible injury has already occurred. Thus, such neonates, whether full-term or premature, are at considerable risk of morbidity and mortality. Abdominal NIRS (aNIRS) may permit early diagnosis, treatment, and monitoring of gastrointestinal hypoxia and hypoperfusion.

Because aNIRS measures a broad anatomic region, it may capture tissue outside the gastrointestinal tract. Therefore, we tested the accuracy of aNIRS for detecting gastrointestinal hypoxia during graded whole-body hypoxia and splanchnic hypoperfusion from superior mesenteric artery (SMA) ligation. We hypothesized that aNIRS would correlate with the portal vein oxyhemoglobin saturation during whole-body hypoxia and with terminal ileum laser-Doppler flowmetry (LDF) during SMA ligation. We also tested whether umbilical and portal vein oxyhemoglobin saturations correlate during hypoxia, because umbilical venous blood gases are clinically available in routine neonatal intensive care.

\section{METHODS}

\section{Animal Preparation}

All procedures were approved by the Animal Care and Use Committee at Johns Hopkins University, and the care and handling of the animals were in accord with the National Institutes of Health guidelines. Full-term, neonatal male piglets (3-5 days old, $1.5-2 \mathrm{~kg}$ ) were anesthetized with $5 \%$ isoflurane and $50 \% / 50 \%$ nitrous oxide/ oxygen for tracheostomy. Mechanical ventilation was used to maintain normocapnea. Isoflurane was decreased to $2 \%$ after tracheostomy, and the femoral artery and the vein were cannulated for arterial blood pressure monitoring and intravenous (IV) administration of medications and fluids. The nitrous oxide was discontinued after placement of the femoral venous catheter. Fentanyl $(10 \mu \mathrm{g} / \mathrm{kg}$ bolus $+10 \mu \mathrm{g} / \mathrm{kg} / \mathrm{h}), 1.5 \%$ isoflurane, and rocuronium $(0.2 \mathrm{mg} / \mathrm{kg} / \mathrm{h})$ were administered for the remainder of the experiment. Piglets received maintenance IV fluids with 5\% dextrose in $0.45 \%$ saline at $4 \mathrm{ml} / \mathrm{kg} / \mathrm{h}$. Arterial blood pressure, heart rate, rectal temperature, and end-tidal $\mathrm{CO}_{2}$ were monitored continuously. Hemoglobin and blood gas data were obtained at time points described below.

\footnotetext{
'Division of Neonatology, Department of Pediatrics, Johns Hopkins University School of Medicine, Baltimore, Maryland; ${ }^{2}$ Department of Anesthesiology and Critical Care Medicine, Johns Hopkins University School of Medicine, Baltimore, Maryland; ${ }^{3}$ Department of Pediatric Surgery, Johns Hopkins University School of Medicine, Baltimore, Maryland. Correspondence: May W. Chen (maywchen@jhmi.edu) 


\section{Graded Hypoxia}

We placed portal and umbilical venous catheters in piglets destined for whole-body hypoxia. The umbilical vein was cannulated through the umbilical stump with a 3.5 Fr umbilical catheter (Utah Medical Products, Midvale, UT) that was advanced 5-6 cm. Then, a midline laparotomy was performed for splenic vein cannulation with a $3.5 \mathrm{Fr}$ catheter, which was advanced into the portal vein. The incision was then sutured closed. An aNIRS probe (Medtronic, INVOS 5100, pediatric NIRS sensor, Medtronic, Minneapolis, MN) was placed over the right lower abdominal quadrant to target the terminal ileum, a region commonly affected by NEC. The right lower quadrant also avoids the bladder, which may become distended from urinary retention during general anesthesia. The INVOS NIRS system uses two wavelengths of near-infrared light at 730 and $810 \mathrm{~nm}$ to measure the concentrations of regional oxygenated and deoxygenated hemoglobin (5). The pediatric sensors have an emitter to distal (deep) detector distance of $40 \mathrm{~mm}$, with a signal penetration depth of $2 \mathrm{~cm}$.

After a 1-h post-operative recovery period, we induced wholebody hypoxia through eight stepwise decreases in the percent of inspired oxygen $\left(\mathrm{FiO}_{2}\right)$ from 21 to $10 \%$. Piglets were exposed to each $\mathrm{FiO}_{2}$ level $(21,18,16,15,14,13,12,11$, and $10 \%)$ for $6 \mathrm{~min}$. At the completion of each 6-min interval, we sampled the portal vein, umbilical vein, and femoral artery blood for the measurement of $\mathrm{pH}$, blood gases, hemoglobin, and $\mathrm{O}_{2}$ saturation (Radiometer ABL Flex 800, Copenhagen, Denmark). We also recorded the aNIRS oxyhemoglobin saturation at the same time points for analysis. We ensured that the aNIRS oxyhemoglobin saturation was stable over $60 \mathrm{~s}$ before recording the data to minimize the risk of artifact. Piglets were euthanized with Beuthanasia-D $(50 \mathrm{mg} / \mathrm{kg}$, Henry Schein Animal Health, Dublin, $\mathrm{OH}$ ), in addition to isoflurane and fentanyl, before postmortem confirmation that the catheters were correctly placed in the umbilical and portal veins.

\section{SMA Ligation}

A separate group of animals underwent SMA ligation. After placing the femoral arterial and venous catheters as described above, we made a midline laparotomy incision. We then identified the terminal ileum and secured a 1-mm diameter laser-Doppler probe (Moor Instruments, Devon, England) to it with liquid adhesive. The incision was sutured closed and an aNIRS probe was placed over the right lower abdominal quadrant, as described above. Finally, we identified the SMA through a left flank retroperitoneal incision and encircled it loosely with 3-0 silk suture.

After a 1-h post-operative recovery period, a baseline LDF value was recorded. We then ligated the SMA by tightening the suture. The post-ligation LDF and aNIRS measurements were recorded every $5 \mathrm{~min}$ for $15 \mathrm{~min}$ and then every $15 \mathrm{~min}$ for $180 \mathrm{~min}$. Piglets were euthanized with Beuthanasia-D $(50 \mathrm{mg} / \mathrm{kg})$, in addition to the isoflurane and fentanyl, after which we confirmed positioning of the ileal laser-Doppler probe and SMA ligature.

\section{Splanchnic-Cerebral Oxygenation Ratio}

A pediatric NIRS probe was placed across the piglets' forehead for cerebral oximetry with cerebral NIRS (cNIRS), during graded hypoxia and SMA ligation. We recorded single cNIRS data points simultaneously with the aNIRS data acquisition at the end of each $\mathrm{FiO}_{2}$ 6-min exposure and at each time point during SMA ligation. The values were then used to calculate the splanchnic-cerebral oxygenation ratio $(\mathrm{SCOR}=\mathrm{aNIRS} / \mathrm{cNIRS})$.

\section{Statistical Analysis}

Data were analyzed with SigmaPlot (v12.3, Systat Software, San Jose, CA), and the graphs were generated with SigmaPlot and GraphPad Prism (v7.02, GraphPad Software Inc., La Jolla, CA). Data are presented as medians and interquartile ranges (IQR). The changes over time were analyzed with the Friedman repeated-measures analysis of ranks test, with post hoc comparison to the baseline value by Dunnett's test. In each piglet subjected to graded hypoxia, linear

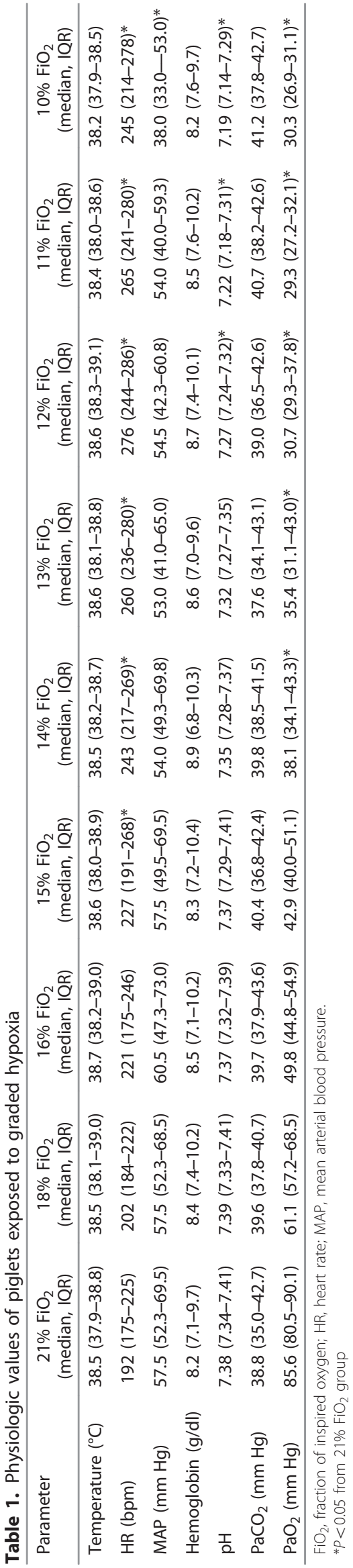




\section{Articles | chen et al.}

regression analysis was performed for aNIRS oximetry values and umbilical vein $\mathrm{O}_{2}$ saturation values, as functions of portal vein $\mathrm{O}_{2}$ saturation. For pooled data, Bland Altman analysis was performed to evaluate the potential bias at low levels of oxygenation that would be clinically relevant. LDF was analyzed as a percentage of the baseline. Statistical significance was set at $P<0.05$.

\section{Sample Size}

We did not have a priori data in our full-term piglet model with graded hypoxia or SMA occlusion. A previous study in premature piglets reported a difference in aNIRS saturations of $6.5 \%$ (standard deviation (SD): 4) between piglets with non-fulminant NEC and those with no NEC (6). Using these estimates, a sample size of seven would reject the null hypothesis that injured and non-injured piglets have similar aNIRS values at an alpha level of 0.05 and power of 0.8 . Because we are studying full-term piglets and we did not test for pathologic evidence of NEC (and thus the effect size may be smaller), we increased the sample size by $40 \%$ to test aNIRS during graded hypoxia.
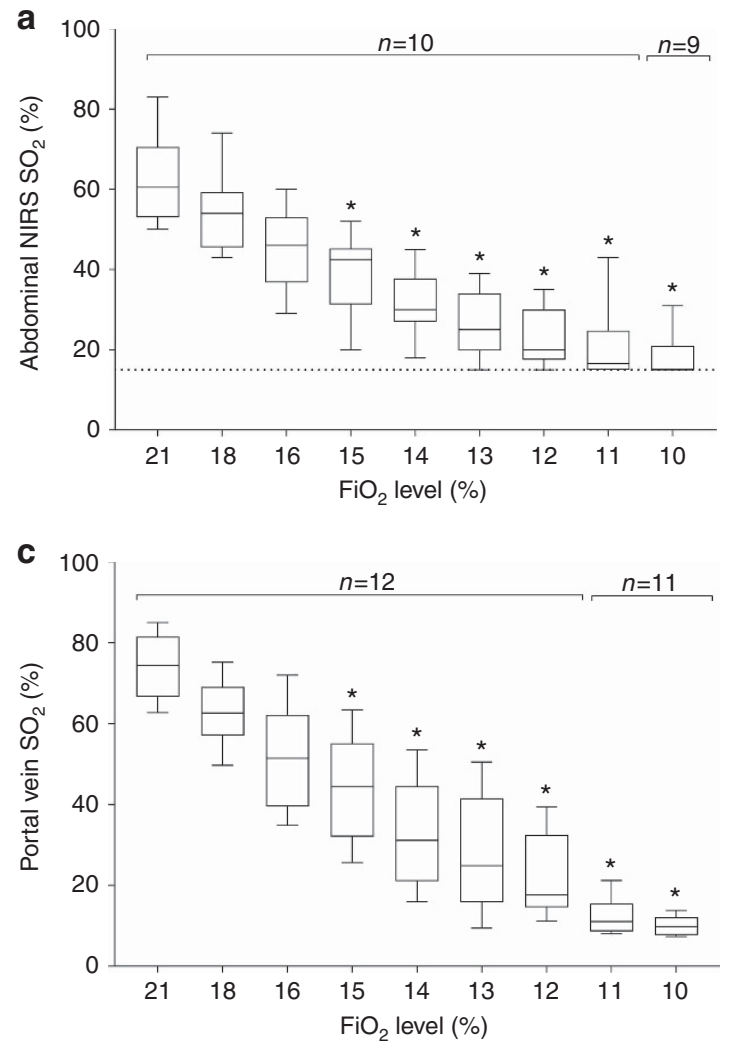

A study in adult rabbits with SMA occlusion and bowel reperfusion reported a difference in the aNIRS tissue oxygenation index (similar to the oxyhemoglobin saturation used in our study) of 8\% (SD: 3.7) (7). With these estimates, a sample size of five would reject the null hypothesis of no difference across time with bowel ischemia and reperfusion at an alpha level of 0.05 and power of 0.8 . We increased the sample size by $60 \%$ in our neonatal piglets to study aNIRS during SMA occlusion.

\section{RESULTS}

Twelve male piglets received graded, whole-body hypoxia and eight male piglets underwent SMA ligation. We monitored aNIRS in 10 piglets during hypoxia and in 8 during SMA ligation. The cNIRS was monitored in eight piglets during hypoxia and in eight during SMA ligation. We could not monitor aNIRS in all piglets, because the aNIRS cable stopped working and then had to be replaced in two piglets.
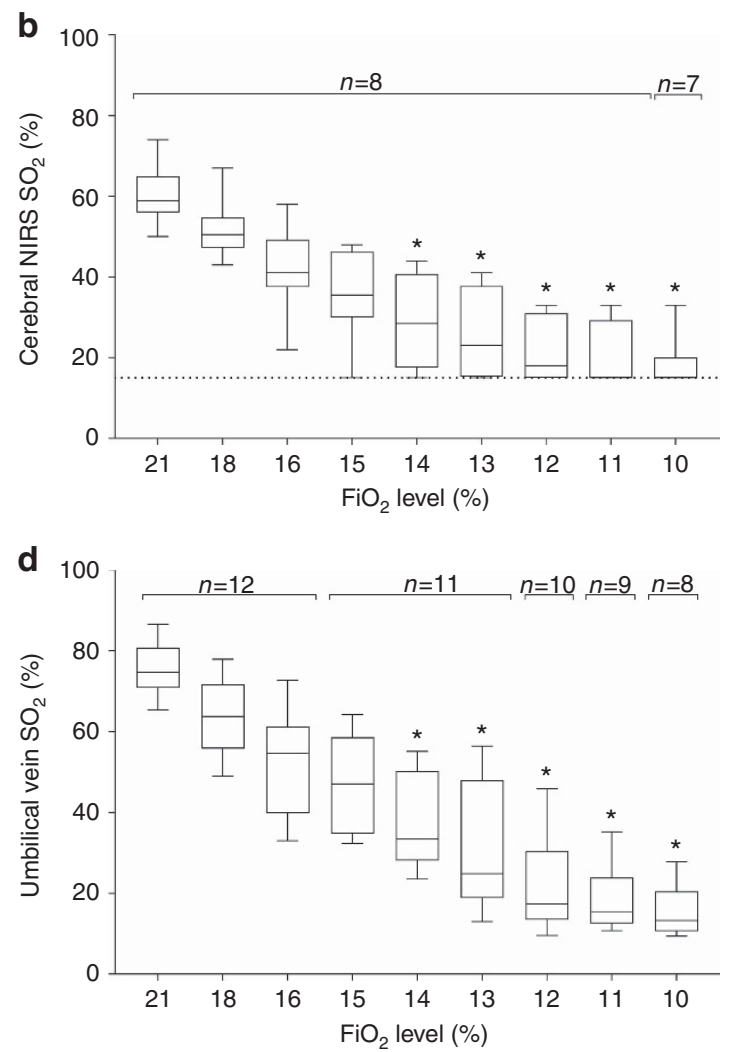

Figure 1. Graded hypoxia in piglets. Oxyhemoglobin saturation decreased during progressive hypoxia, as measured by abdominal near-infrared spectroscopy (NIRS) (a; $P<0.001)$, cerebral NIRS $(\mathbf{b} ; P<0.001)$, portal vein $(\mathbf{c} ; P<0.001)$, and umbilical vein $(\mathbf{d} ; P<0.001)$. The abdominal NIRS and portal vein saturations first detected a significant decrease in oxyhemoglobin saturation between $\mathrm{FiO}_{2} 21 \%$ and $\mathrm{FiO}_{2} \quad 15 \%$. Data were analyzed by Friedman repeated measures analysis of ranks, with a post hoc Dunnett's test. ${ }^{*} P<0.05$ vs. $\mathrm{FiO}_{2} 21 \%$ by Dunnett's test. $\mathrm{SO}_{2}$, oxyhemoglobin saturation.

Figure 2. Paired correlations in oxyhemoglobin saturation $\left(\mathrm{SO}_{2}\right)$ levels measured in the portal vein (PV) and by abdominal near-infrared spectroscopy (aNIRS), during graded hypoxia. Pearson correlation coefficients were high in each of 10 piglets $(\mathbf{a}-\mathbf{j})$. The solid line represents the regression line, and the equation is given in each panel. The dotted line represents the line of identity. For the 10 piglets, the mean and $95 \%$ confidence limits of the aNIRS intercept (k) and the regression slope (I) are shown to differ from zero and one, respectively. When the data from the 10 piglets were pooled $(\mathbf{m})$, the correlation coefficient was also high (0.92). The mean bias determined with a Bland Altman plot (n) was $-0.3 \%$ and the limits of agreement $( \pm 1.96 \mathrm{SD})$ ranged from -19.0 to $18.4 \%$. Dashed lines represent the mean difference and the limits of agreement. 


\section{Graded Hypoxia}

Table 1 lists the physiologic values during graded hypoxia.

The partial pressure of arterial oxygen $\left(\mathrm{PaO}_{2}\right)$ decreased from
$85 \mathrm{~mm} \mathrm{Hg}$ during room air to $30 \mathrm{~mm} \mathrm{Hg}$ at $\mathrm{FiO}_{2} \quad 10 \%$. Temperature decreased slightly at an $\mathrm{FiO}_{2}$ of $10 \%$, heart rate increased significantly at $\mathrm{FiO}_{2}$ levels below $16 \%$, mean arterial
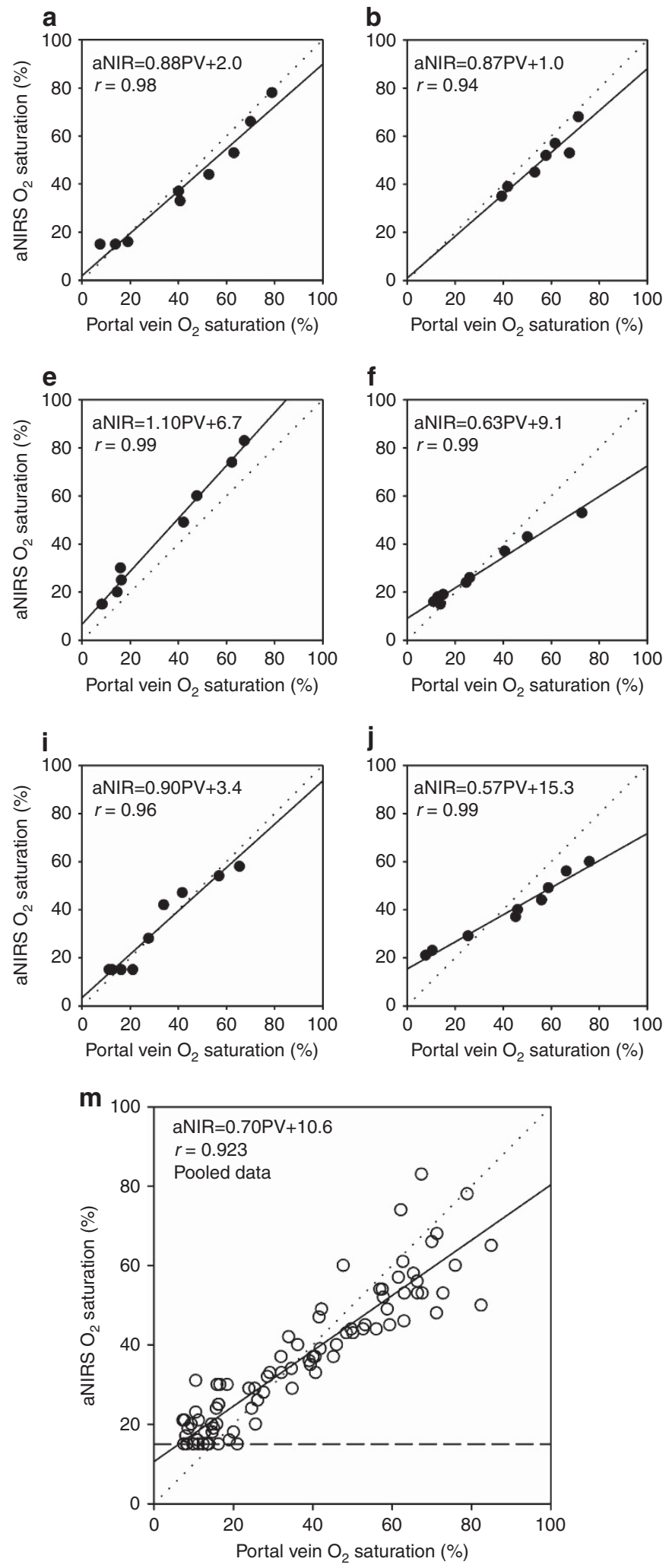
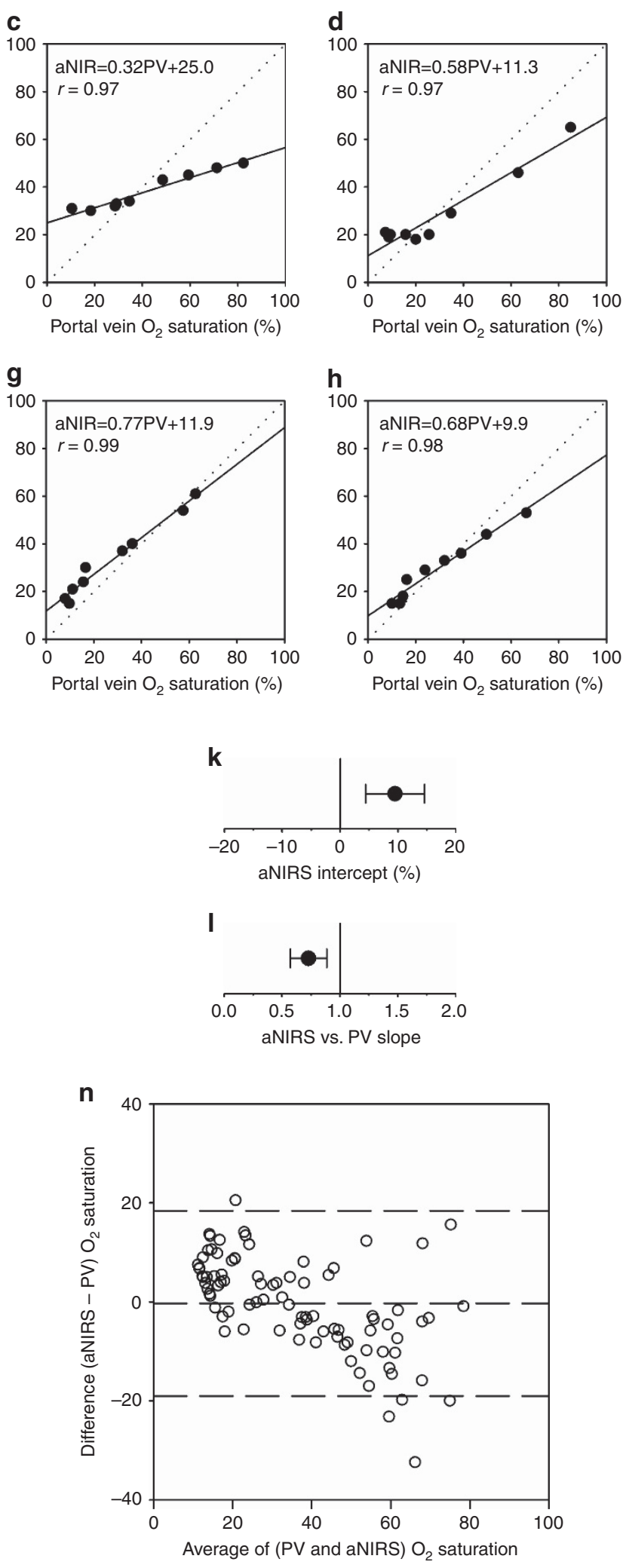


\section{Articles | chen et al.}

blood pressure decreased at an $\mathrm{FiO}_{2}$ of $10 \%$, and the $\mathrm{pH}$ decreased at $\mathrm{FiO}_{2}$ levels below $13 \%$. Hemoglobin $(P=0.353)$ and $\mathrm{PaCO}_{2}(P=0.293)$ levels did not differ as $\mathrm{FiO}_{2}$ decreased.

The oxyhemoglobin saturation, as measured by aNIRS $(n=10, P<0.001)$, cNIRS $(n=8, P<0.001)$, portal vein $(n=12, P<0.001)$, and umbilical vein $(n=12, P<0.001)$, decreased with progressive hypoxia (Figure 1). Significant decreases in aNIRS and portal vein saturations from the baseline values were first identified at an $\mathrm{FiO}_{2}$ of $15 \%$ $\left(P<0.05\right.$ for both), which corresponded to a $\mathrm{PaO}_{2}$ of $43 \mathrm{~mm} \mathrm{Hg}$. Likewise, the umbilical vein and cNIRS saturations first differed from baseline at an $\mathrm{FiO}_{2}$ of $14 \%$ $(P<0.05$ for both). Some piglets did not display NIRS readings at $\mathrm{FiO}_{2} 10 \%(n=1)$, and some piglets' portal $(n=1)$ and umbilical catheters $(n=4)$ did not have blood return at $\mathrm{FiO}_{2} 11 \%$ and lower.

For each of the 10 piglets in which we obtained aNIRS during hypoxia, the relationship of aNIRS tissue oxyhemoglobin saturation to portal vein oxyhemoglobin saturation was highly linear, with correlation coefficients ranging from 0.94 to 0.99 (Figure $2 \mathbf{a}-\mathbf{j}$ ). The average regression coefficient was 0.73 (95\% confidence interval (CI): 0.57, 0.89), which differed significantly from one $(P<0.004)$ by $t$-test (Figure 21). The aNIRS-intercept averaged 9.5\% (CI: 4.4, 14.6), which differed significantly from zero $(P<0.0025)$ by $t$-test (Figure $2 \mathbf{k}$ ).
When we pooled the data from 10 piglets, the correlation coefficient was 0.923 , the regression slope was 0.70 , and the aNIRS intercept was $10.6 \%$ (Figure $2 \mathrm{~m}$ ). The mean bias determined with a Bland Altman plot (Figure 2n) was $-0.3 \%$, and the limits of agreement $( \pm 1.96 \mathrm{SD})$ ranged from -19.0 to $18.4 \%$. Although the mean bias of $-0.3 \%$ is not clinically meaningful, it should be noted that the bias varied significantly with the level of oxygenation (bias $=10.1 \% \quad-0.29$ average $\mathrm{O}_{2}$ saturation; $r=0.60$; $P<0.001)$. The positive bias at low levels of oxygenation and negative bias at high levels of oxygenation (Figure 2n) are consistent with the positive aNIRS intercept and the slope $<1$ in the regression of aNIRS with $\mathrm{PV} \mathrm{O}_{2}$ saturation (Figure $2 \mathbf{k}, \mathbf{l})$. Part of the positive bias may be related to the fact that the Medtronic NIRS oximetry readout truncates at values $<15 \%$.

The tissue oxyhemoglobin saturation measured by aNIRS represents a mixture of arterial and venous blood. When the relationship of aNIRS oxyhemoglobin saturation was compared to arterial oxyhemoglobin saturation during graded hypoxia, a linear relationship was obtained with a correlation coefficient of 0.93 (Figure 3a). In this case, however, Bland Altman analysis indicated a negative bias averaging 23.6\%, over the entire range of arterial saturations (Figure $3 \mathbf{b}$ ). The lower aNIRS reading at each arterial $\mathrm{O}_{2}$ saturation reflects the
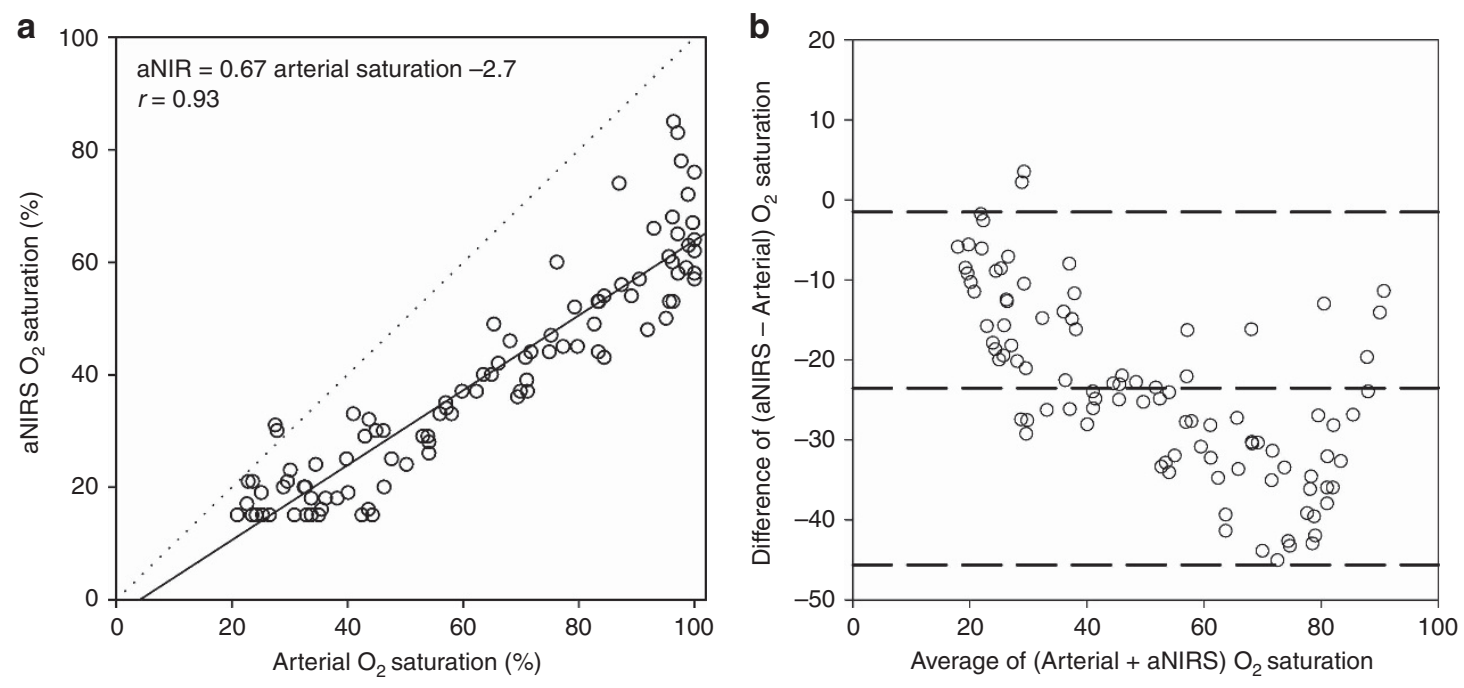

Figure 3. Paired correlation in arterial oxyhemoglobin saturation and abdominal near-infrared spectroscopy (aNIRS) oxyhemoglobin saturation during graded hypoxia (a). The solid line represents the regression line. The dotted line represents the line of identity. Data pooled from 10 piglets indicates that aNIRS measurements underestimate arterial $\mathrm{O}_{2}$ saturation. Likewise, the Bland Altman plot (b) demonstrated a significant bias of aNIRS against arterial oxyhemoglobin saturation. Dashed lines represent the mean difference $(-23.6 \%)$ and the limits of agreement $(-45.7,-1.5)$.

Figure 4. Paired correlations in oxyhemoglobin saturation $\left(\mathrm{SO}_{2}\right)$ levels measured in the portal vein and the umbilical vein (UV) during graded hypoxia. Pearson correlation coefficients were high in each of the 12 piglets $(\mathbf{a}-\mathbf{I})$. The solid line represents the regression line, and the equation is given in each panel. The dotted line represents the line of identity. For the 12 piglets, the mean and $95 \%$ confidence limits of the umbilical vein $\mathrm{O}_{2}$ saturation intercept $(\mathbf{m})$ are shown to differ from zero, whereas, the regression slope (n) did not significantly differ from one. When the data from the 12 piglets were pooled (o), the correlation coefficient was also high (0.95) and the Bland Altman plot (p) did not reveal a meaningful bias of UV against PV saturation. Dashed lines represent the mean difference (2.2\%) and the limits of agreement (-10.8\%, 15.2\%). 
small compartment of arterialized blood, relative to the capillary and venous compartments in the tissue monitored by aNIRS. The bias was less negative at low oxygenation, presumably because the difference between the arterial and venous $\mathrm{O}_{2}$ saturation shrinks during hypoxia (bias $=-6.2 \%-$ 0.34 average $\mathrm{O}_{2}$ saturation; $r=0.68 ; P<0.001$ ).
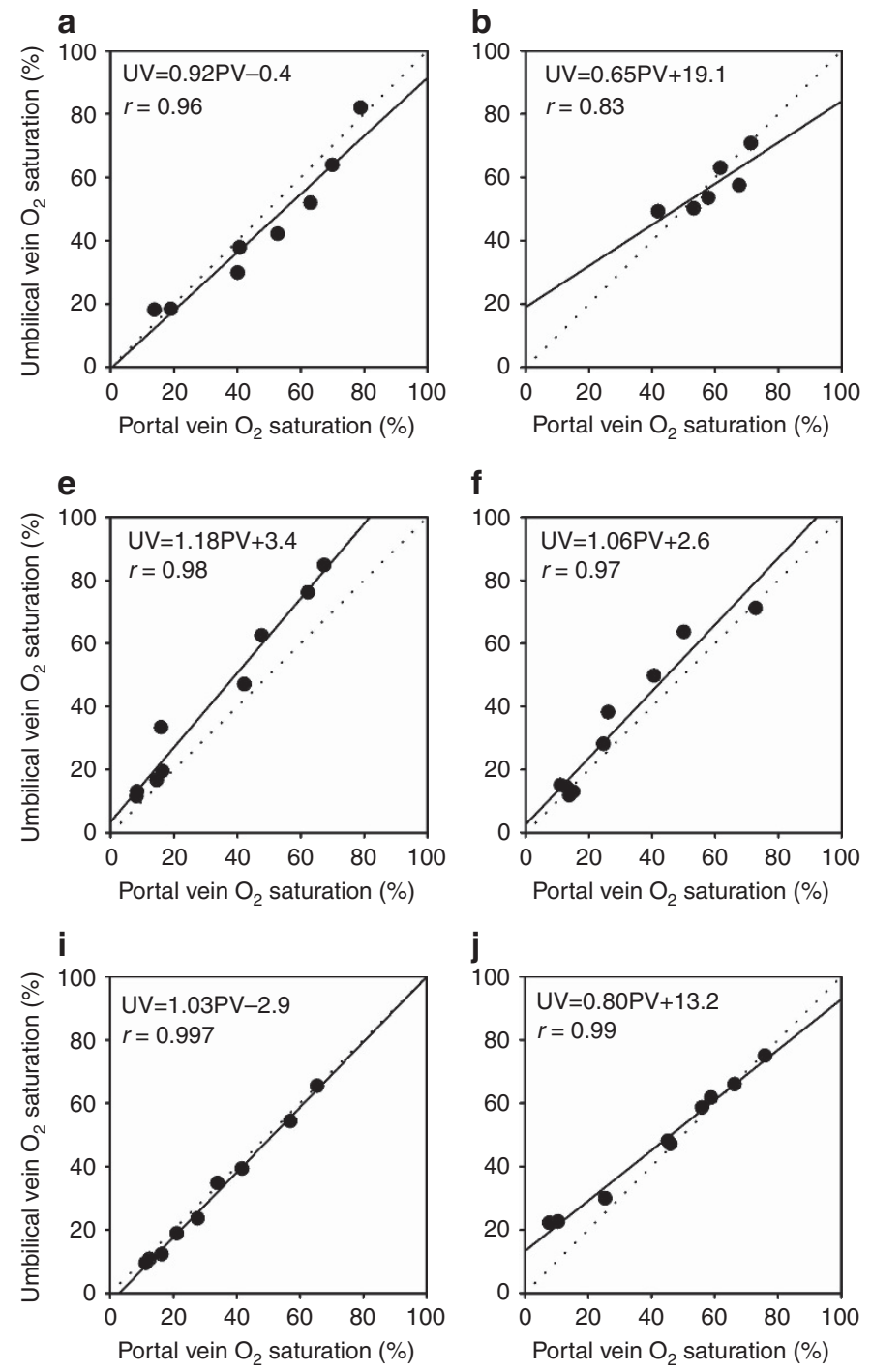

j

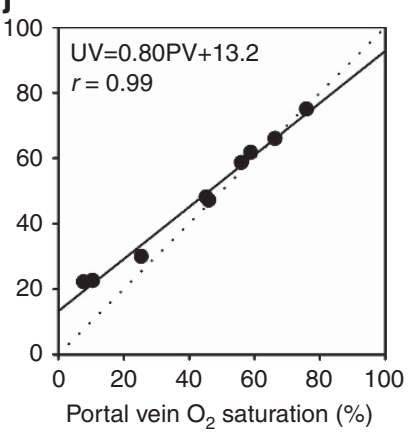

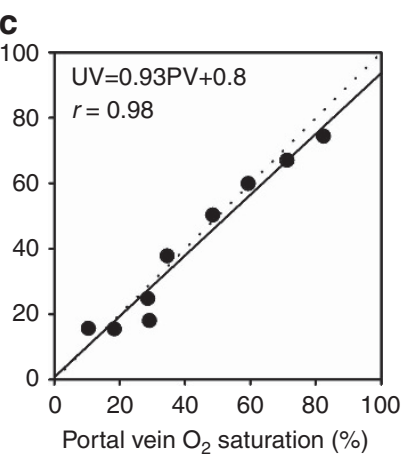

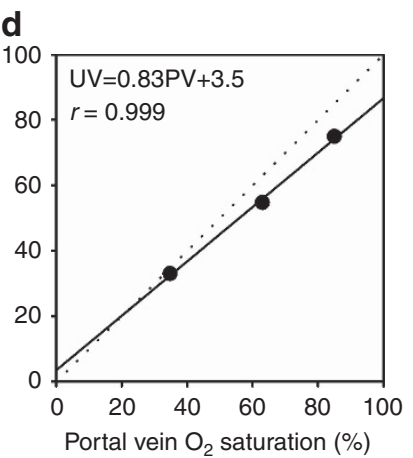

g

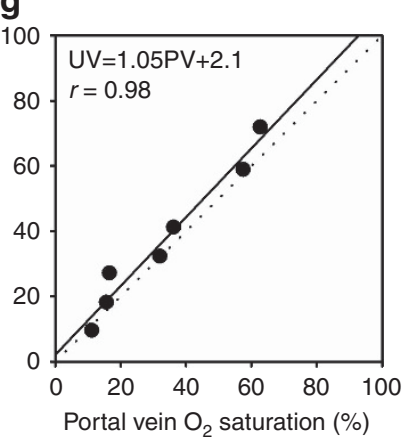

h

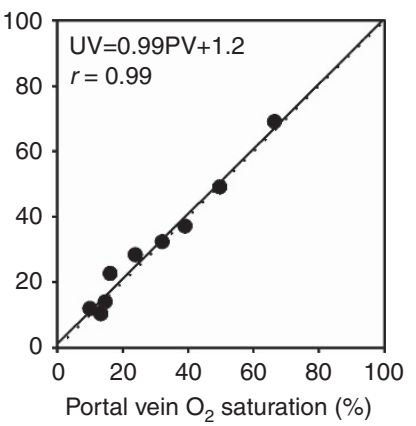

k

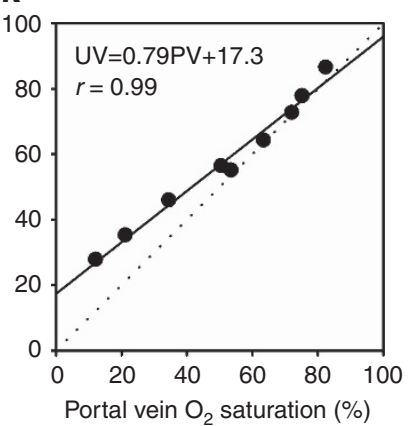

I

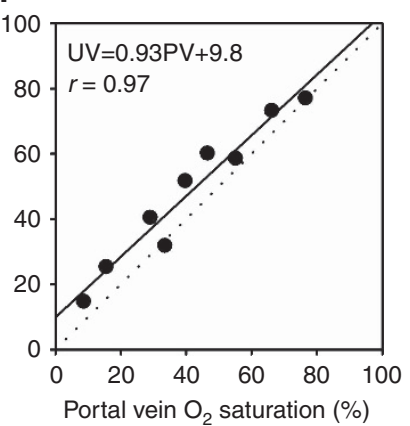

m

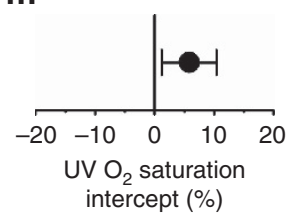

n

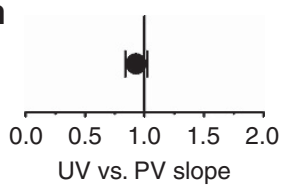

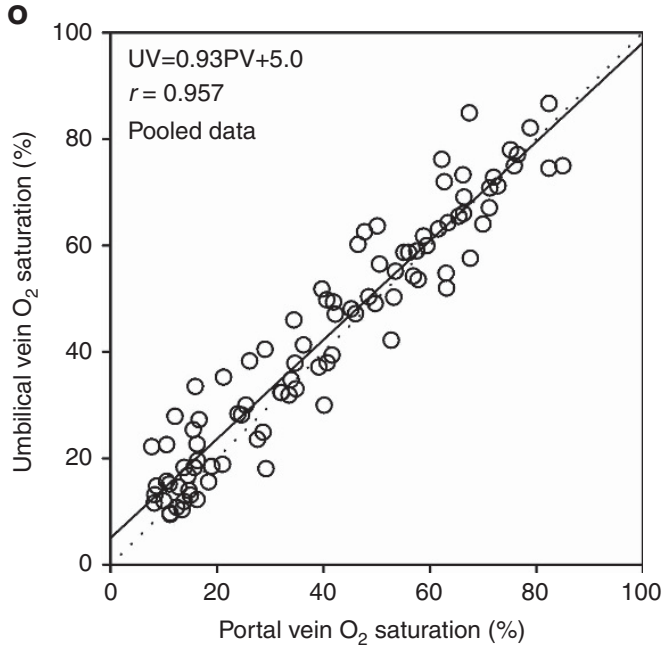

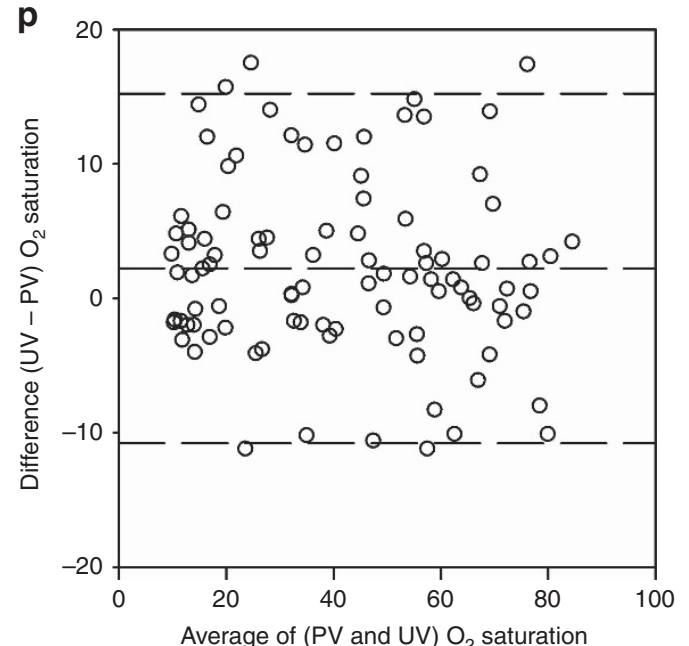




\section{Articles | chen et al.}

We were also interested in determining whether the umbilical vein oxyhemoglobin saturation could serve as a surrogate for the portal vein oxyhemoglobin saturation. The relationship between portal and umbilical venous oxyhemoglobin saturations was also highly linear, with correlation coefficients ranging from 0.83 to 0.99 among the 12 piglets in which both measurements were obtained (Figure 4a-1). The average regression coefficient was 0.93 (CI: $0.84,1.02$ ), which was not significantly different from one $(P=0.12)$ by $t$-test (Figure $4 \mathbf{n})$. The umbilical venous intercept averaged $5.8 \%$ (CI: 1.2, 10.4), which differed significantly from zero $(P<0.02)$ by $t$-test $($ Figure $4 \mathrm{~m})$. When we pooled the data from the 12 piglets, the correlation coefficient was 0.957 , the regression slope was 0.93 , and the umbilical venous intercept was 5.0\% (Figure 4o). The Bland Altman plot of the umbilical vein oxyhemoglobin saturation against the portal vein oxyhemoglobin saturation (Figure 4p) indicted a mean bias of 2.2\% (limits of agreement $-10.8 \%, 15.2 \%)$, and this small bias did not significantly vary with the level of oxygenation $(P=0.36)$.

\section{SMA Ligation}

Table 2 lists the physiologic values during the $3 \mathrm{~h}$ of SMA ligation. During SMA ligation, mean arterial pressure increased significantly from the baseline during the initial $15 \mathrm{~min}$ and then returned to the baseline. In the last $90 \mathrm{~min}$ of ligation, arterial $\mathrm{pH}$ and hemoglobin concentration significantly decreased, whereas temperature, heart rate, $\mathrm{PaCO}_{2}$, and $\mathrm{PaO}_{2}$ were not significantly changed from baseline levels.

With SMA ligation, aNIRS saturation $(P<0.001)$ and LDF $(P<0.001)$ decreased acutely by $5 \mathrm{~min}$ after ligation, and remained low through 180 min (Figure 5a,b). However, at $5 \mathrm{~min}$ and beyond, the aNIRS and LDF values did not vary over the duration of SMA ligation $(P=0.299$ for aNIRS; $P=0.511$ for LDF). The cNIRS saturations did not differ significantly from baseline (Figure $5 \mathrm{c}$ ). The time-averaged change in the aNIRS reading among the eight piglets correlated with time-averaged change in LDF $(r=0.87$; Figure 5d). One piglet did not have the 5- and 10-min data because the time points were missed during the catheter manipulation. Two piglets died before completion of the 180min post-ligation period.

\section{SCOR}

The SCOR $(=$ aNIRS/cNIRS $)$ did not differ between $\mathrm{FiO}_{2}$ levels with progressive, graded hypoxia $(P=0.876)$. However, the SCOR values decreased significantly after SMA ligation $(P<0.0001$; Figure 6).

\section{DISCUSSION}

Our findings indicate that aNIRS detects gastrointestinal hypoxia during whole-body hypoxia as well as during splanchnic hypoperfusion caused by SMA ligation in neonatal, full-term piglets. Oxyhemoglobin saturations determined by aNIRS and portal vein significantly correlated in a linear fashion during graded hypoxia, showing that aNIRS reflects gastrointestinal oxygenation over a wide range of

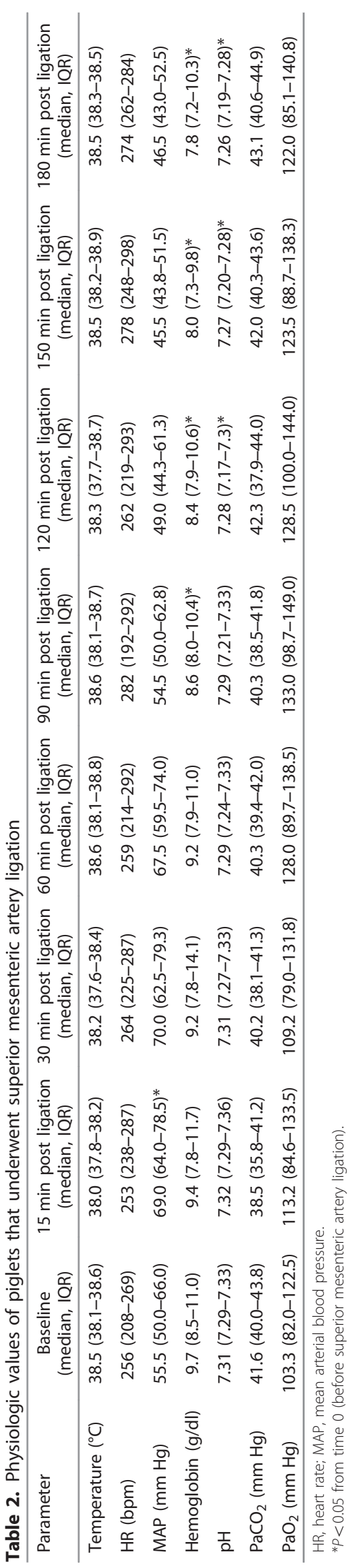




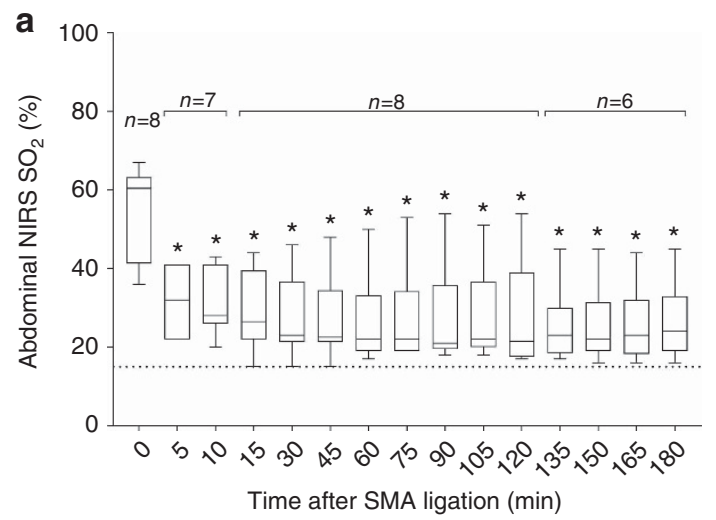

b
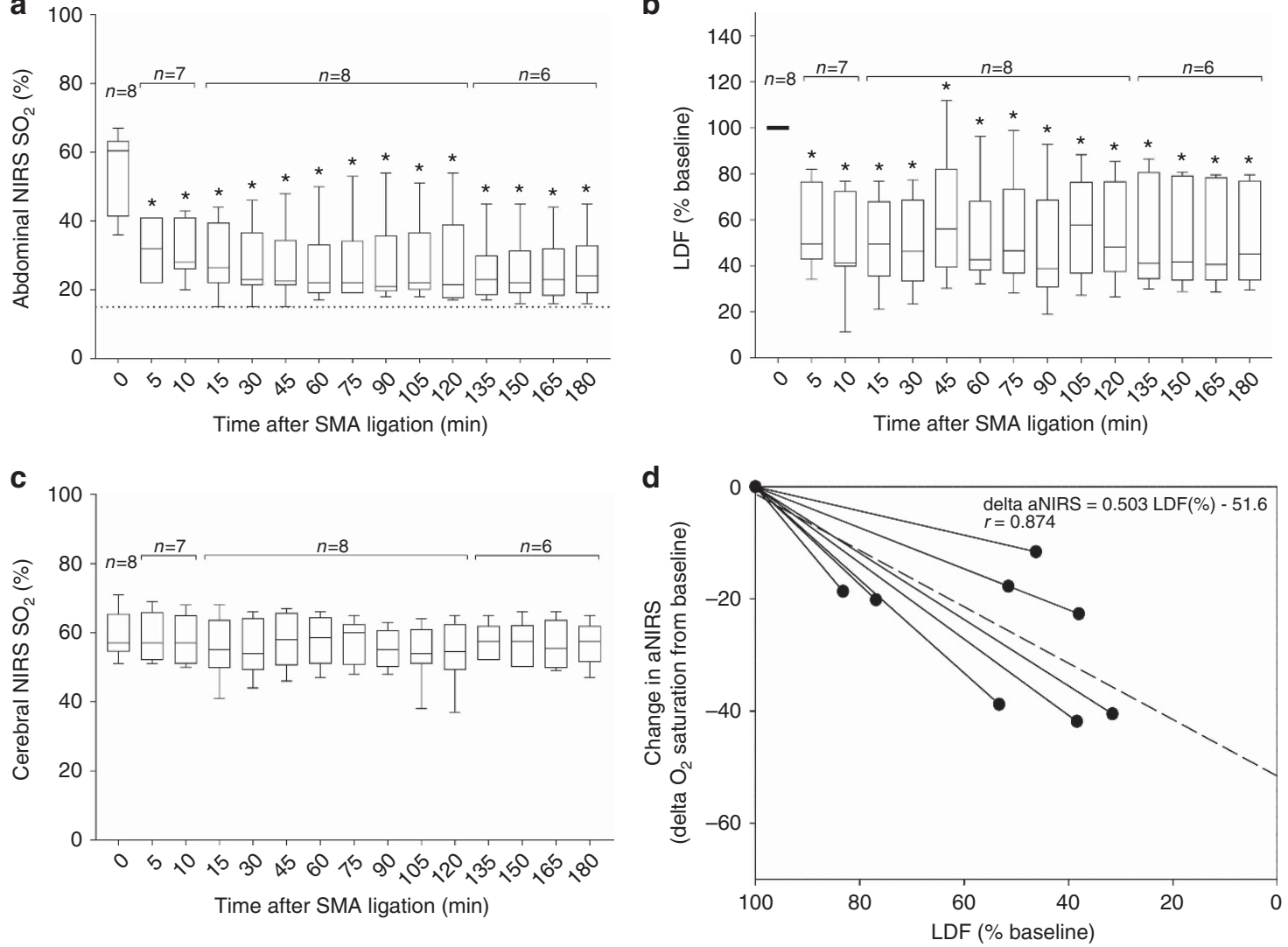

Figure 5. After superior mesenteric artery (SMA) ligation, the abdominal near-infrared spectroscopy (a, NIRS) and ileal laser-Doppler flow (b, LDF) measures decreased acutely, whereas, the cerebral NIRS (c) remained unchanged. The abdominal NIRS $(P=0.299)$ and LDF $(P=0.511)$ did not differ during the period of 5-180 min after SMA ligation, indicating that gastrointestinal perfusion did not improve with time. Time 0 is before SMA ligation. Data were analyzed by Friedman repeated-measures analysis of ranks, with post hoc Dunnett's test. ${ }^{*} P<0.05$ vs. time 0 by Dunnett's test. (d) When data from all time points were averaged for each piglet, the change in aNIRS from baseline correlated with the change in LDF, expressed as a percent of baseline. Dashed line indicates the overall regression line for the pooled data: delta aNIRS $=-51.6+0.503$ LDF $(\%)$; $r=0.874$.
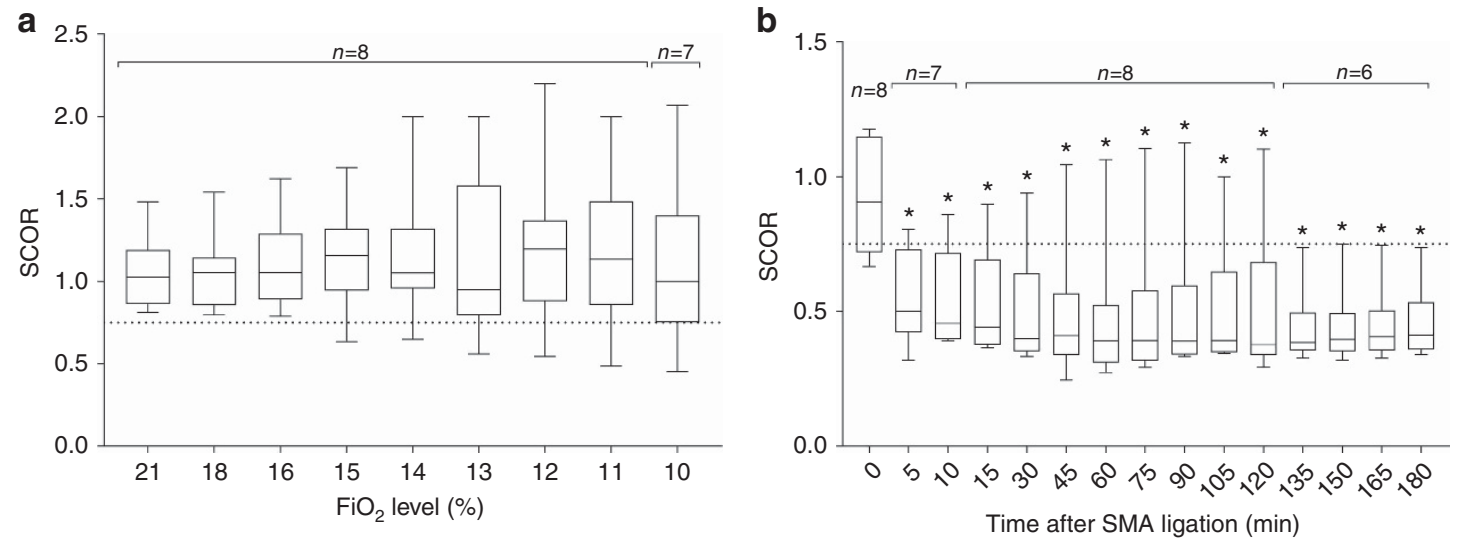

Figure 6. The splanchnic cerebral oxygenation ratio (SCOR; abdominal NIRS/cerebral NIRS) did not differ during graded hypoxia (a; $P=0.876)$, but decreased after superior mesenteric artery (SMA) ligation (b). Time 0 is before SMA ligation. ${ }^{*} P<0.05$ from time 0 by Dunnett's test. 
hypoxia. Inaccuracies in the aNIRS readings occurred when portal vein saturations approached $15 \%$, which is the lower boundary of NIRS. Nevertheless, the correlation remained tight at the low levels of tissue oxygenation, that would be of most importance in a clinical setting for flagging impending ischemia. Furthermore, inducing ischemia by SMA ligation caused terminal ileal blood flow and aNIRS to decrease acutely and remain low through $180 \mathrm{~min}$. Specifically, the aNIRS oximetry decreased from approximately 60 to $30 \%$, which implies that the arterio-venous $\mathrm{O}_{2}$ content difference nearly doubled. A near doubling of the $\mathrm{O}_{2}$ extraction would be expected to offset the observed 50\% decrease in blood flow and help maintain $\mathrm{O}_{2}$ uptake in the gut. Collectively, our piglet study supports the use of aNIRS to detect neonatal gastrointestinal hypoxia/ischemia.

During graded hypoxia, we found that the regression of aNIRS oximetry with portal vein $\mathrm{O}_{2}$ saturation, although highly linear, had a positive intercept and a slope $<1$. One consideration is that aNIRS measures an average tissue oxyhemoglobin concentration that comprises arterial and venous compartments. The arterialized vascular compartment is smaller than the capillary and venous vascular compartments. Consequently, the aNIRS signal was consistently lower than the measured arterial oxyhemoglobin saturation during graded hypoxia (Figure 3). In contrast, the admixture of arterialized blood in the oximetry signal presumably accounts for the positive intercept on the aNIRS axis, when correlated with a purely venous blood sample (Figure 2). The observation that the slope of the relationship of aNIRS with portal vein oxyhemoglobin saturation is $<1$ suggests that the weighting of the arterial and venous compartments in the oximetry signal varies during hypoxia, such that the arterial compartment is more heavily weighted during severe hypoxia. This interpretation would be consistent with active vasodilation in the arterial compartment, and perhaps reflex constriction in the venous compartment during severe hypoxia. Other interpretations include non-uniform blood flow or metabolism within the gastrointestinal tract subtended by the NIRS optodes relative to the regions drained by the portal vein. Another consideration is that some nongastrointestinal tissue is captured by aNIRS.

The portal vein is formed from the union of the splenic and superior mesenteric veins, which carry blood from the intestines. Because portal vein sampling is not feasible in routine clinical practice, aNIRS might serve as a useful surrogate for measuring gastrointestinal oxygenation, given its close correlation with portal vein oxyhemoglobin saturation. Moreover, the umbilical and portal vein saturations correlated during hypoxia, in a highly linear manner, with a slope not different from one and with only a small positive intercept of the umbilical vein $\mathrm{O}_{2}$ saturation (Figure 4). Furthermore, the Bland Altman plot did not reveal a clinically meaningful bias between the two measurements over the entire range of oxygenation. Another study in piglets also reported a correlation between umbilical vein and portal vein $\mathrm{O}_{2}$ saturations (8). Many critically ill neonates have umbilical catheters. Using a combination of aNIRS and umbilical vein oxyhemoglobin saturations might aid in the early detection of gastrointestinal hypoxia. The fact that the relationship between aNIRS and portal vein $\mathrm{O}_{2}$ saturation was highly linear in every animal, implies that the aNIRS can be used to track trends in tissue oxygenation over time within a given patient. If, on the other hand, only one or infrequent aNIRS measurements can be obtained from a patient, the absolute value from aNIRS should still represent a value that is close to the actual portal vein $\mathrm{O}_{2}$ saturation. This conclusion is supported by the pooled data in Figure $2 \mathrm{~m}$, which shows that most of the data points are close to the line of identity, especially at portal vein $\mathrm{O}_{2}$ saturations $<50 \%$.

We manipulated gastrointestinal perfusion by ligating the SMA. Though we anticipated that the circulation from the celiac and the gastro-duodenal arteries would permit ileal blood flow to recover, we instead observed consistently low perfusion of $\sim 50 \%$ of baseline for $3 \mathrm{~h}$. This observation suggests that collateral circulation from the celiac and gastroduodenal arteries limit gastrointestinal ischemia and that this collateral flow is already present within $5 \mathrm{~min}$ post ligation. The aNIRS signal was relatively stable through the $3 \mathrm{~h}$ of ischemia, a finding that also corresponds to a stable level of hypoperfusion.

Our findings are in agreement with other preclinical studies that have compared NIRS to gastrointestinal perfusion. NIRS correlated with SMA blood flow in abdominal compartment syndrome (9) and hemorrhage models (10). Adult rabbits had decreased liver oxygenation with SMA occlusion (7). Moreover, lower aNIRS correlated with the development of NEC in premature piglets $(6,11)$. In our model, a significant correlation was evident between the change in aNIRS and the decrease in the perfusion from baseline.

We calculated the SCOR because of growing interest in using splanchnic-to-cerebral oxygenation ratios to detect abdominal hypoxia. The SCOR decreased from a median of $\sim 0.9$ to below 0.5 , after SMA ligation. A threshold of 0.75 has been proposed as a marker of intestinal ischemia (12). Fewer than $25 \%$ of the piglets had a baseline below 0.7 , whereas, fewer than $25 \%$ had a SCOR above 0.7 during SMA ligation. Thus, our data support a threshold of $\sim 0.7$. We did not confirm pathologic intestinal ischemia in this study, and thus, cannot determine whether SCOR was associated with pathologic injury. In contrast, the absence of a significant change of SCOR during worsening hypoxia in our model is likely due to the global delivery of hypoxia to both the vascular beds. In the literature, studies that evaluated SCOR used regional rather than global injury (12-14). Moreover, our piglets were full-term gestation, and it is possible that the cerebral and splanchnic vasodilation response to hypoxia in premature neonates with NEC may differ from that of the full-term gestation neonates.

The tachycardia, hypotension, and acidosis that developed with progressive hypoxia and with prolonged SMA ligation reflect the expected hemodynamic and physiologic changes that occur with tissue hypoxia and hypoperfusion. Although rectal 


\section{Abdominal NIRS in a model of GI hypoxia Articles}

temperature declined from 38.5 to $38.2^{\circ} \mathrm{C}$ in piglets that received graded hypoxia, these temperatures are within the range of swine normothermia. In addition, the decrease in hemoglobin levels during SMA ligation may have been secondary to intestinal capillary fluid absorption from low capillary hydrostatic pressure, which can cause hemodilution.

Options for long-term, noninvasive gastrointestinal monitoring are needed for both premature and full-term neonates, given the risk of early- or late-onset NEC (15). Absolute tissue oxygenation levels $\leq 56 \%$ may be associated with an increased risk of NEC (16). The safety and feasibility of neonatal aNIRS are well established (17-19). With this technique, abdominal oxygen desaturation can be detected at least $24-48 \mathrm{~h}$ before NEC is diagnosed $(3,16,17)$. Importantly, aNIRS readings have been shown to improve after $24 \mathrm{~h}$ of bowel rest and antibiotics (14).

Our study had several limitations. We did not include a sham control group that received neither hypoxia nor SMA ligation. We chose to conduct paired (within pig) comparisons during the course of progressive hypoxia or during SMA ligation to examine temporal changes in intestinal oxyhemoglobin saturation and blood flow within the same animal. This approach is similar to observing the physiologic trends in a neonate during clinical practice. Investigators who recorded the data during graded hypoxia or SMA ligation were not blinded to treatment. The sample size was small in our pilot study, but we will use the data to design future studies of piglet gastrointestinal ischemia. Finally, we did not study the intestinal pathology to confirm NEC or other signs of cellular ischemia. We also did not examine the effects of partial splanchnic ischemia or hypoperfusion or hypoxia-ischemia, which are common clinical scenarios in neonatology.

\section{CONCLUSION}

Our pilot study in full-term, neonatal piglets supports the use of aNIRS to detect gastrointestinal hypoxia or disruptions in intestinal perfusion. Using both aNIRS and umbilical venous oxyhemoglobin saturation may contribute to early identification of neonates with gastrointestinal hypoxia, before irreversible injury occurs.

\section{STATEMENT OF FINANCIAL SUPPORT}

Support was provided by $\mathrm{NIH}$ grants K08NS080984 (J.K.L.) and R01NS060703 (R.C.K.) and an American Heart Association Grant-in-Aid (J.K.L.). D.J.H. is supported by R01GM078238 and R01DK083752.

Disclosure: J.K.L. was a paid advisory board member for Medtronic. J.K.L. also received a research grant from Medtronic for a separate clinical study on cerebrovascular autoregulation. This arrangement has been reviewed and approved by the Johns Hopkins University in accordance with its conflict of interest policies. Medtronic did not provide any support (financial or other) or have any input on the study design, data acquisition, or data interpretation for the current piglet study on gastrointestinal hypoxia and hypoperfusion. The remaining authors declare no conflict of interest.

\section{REFERENCES}

1. Wolfberg AJ, du Plessis AJ. Near-infrared spectroscopy in the fetus and neonate. Clin Perinatol 2006;33:707-28.

2. Mittnacht AJC. Near infrared spectroscopy in children at high risk of low perfusion. Curr Opin Anaesthesiol 2010;23:342-7.

3. Cerbo RM, Cabano R, Di Comite A, Longo S, Maragliano R, Stronati M. Cerebral and somatic rSO2 in sick preterm infants. J Matern Neonatal Med 2012;25 (Suppl 4): 89-92.

4. Bailey SM, Mally PV. Review of splanchnic oximetry in clinical medicine. J Biomed Opt. 2016;21:91306.

5. Ortmann LA, Fontenot EE, Seib PM, Eble BK, Brown R, Bhutta AT. Use of near-infrared spectroscopy for estimation of renal oxygenation in children with heart disease. Pediatr Cardiol 2011;32:748-53.

6. Zamora IJ, Stoll B, Ethun CG, et al. Low abdominal NIRS values and elevated plasma intestinal fatty acid-binding protein in a premature piglet model of necrotizing enterocolitis. PLoS ONE 2015;10: e0125437.

7. Vanderhaegen J, Dehing L, Naulaers G, et al. Use of the liver tissue oxygenation index as a noninvasive parameter of intestinal ischemia in rabbits. World J Surg 2007;31:2359-62.

8. Nowicki PT, Stonestreet BS, Hansen NB, Yao AC, Oh W. Gastrointestinal blood flow and oxygen consumption in awake newborn piglets : effect of feeding. Am J Physiol 1983;245:G697-702.

9. Varela JE, Cohn SM, Giannotti GD, et al. Near-infrared spectroscopy reflects changes in mesenteric and systemic perfusion during abdominal compartment syndrome. Surgery 2001;129: 363-70.

10. Cohn SM, Varela JE, Giannotti G, et al. Splanchnic perfusion evaluation during hemorrhage and resuscitation with gastric near-infrared spectroscopy. J Trauma 2001;50:629-35.

11. Gay AN, Lazar DA, Stoll B, et al. Near-infrared spectroscopy measurement of abdominal tissue oxygenation is a useful indicator of intestinal blood flow and necrotizing enterocolitis in premature piglets. J Pediatr Surg 2011;46:1034-40.

12. Fortune PM, Wagstaff M, Petros A. Cerebro-splanchnic oxygenation ratio (CSOR) using near infrared spectroscopy may be able to predict splanchnic ischaemia in neonates. Intensive Care Med 2001;27: 1401-7.

13. Zabaneh RN, Cleary JP, Lieber CA. Mesentric oxygen saturations in premature twins with and without necrotizing enterocolitis. Pediatr Crit Care Med 2011;12:e404-6.

14. Stapleton GE, Eble BK, Dickerson HA, Andropoulos DB, Chang AC Mesenteric oxygen desaturation in an infant with congenital heart disease and necrotizing enterocolitis. Tex Heart Inst J 2007;34: 442-4.

15. Li Q-Y, An Y, Liu L, et al. Differences in the clinical characteristics of early- and late-onset necrotizing enterocolitis in full-term infants: a retrospective case-control study. Sci Rep 2017;7:43042.

16. Patel AK, Lazar DA, Burrin DG, et al. Abdominal near-infrared spectroscopy measurements are lower in preterm infants at risk for necrotizing enterocolitis. Pediatr Crit Care Med 2014;15:735-41.

17. Cortez J, Gupta M, Amaram A, Pizzino J, Sawhney M, Sood BG. Noninvasive evaluation of splanchnic tissue oxygenation using nearinfrared spectroscopy in preterm neonates. J Matern Neonatal Med 2011;24:574-82.

18. Schat TE, van der Laan ME, Schurink M, et al. Abdominal near-infrared spectroscopy in preterm infants: a comparison of splanchnic oxygen saturation measurements at two abdominal locations. Early Hum Dev 2014;90:371-5.

19. McNeill S, Gatenby JC, McElroy S, Engelhardt B. Normal cerebral, renal and abdominal regional oxygen saturations using near-infrared spectroscopy in preterm infants. J Perinatol 2011;31:51-7. 